\title{
Factors associated with engagement in online self-help programs among people with suicidal thoughts
}

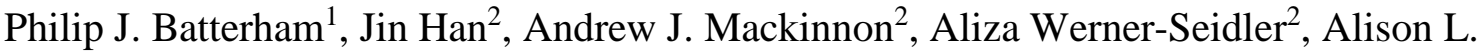
Calear $^{1}$, Quincy Wong ${ }^{2}$, Michelle Torok ${ }^{2}$ \& Helen Christensen ${ }^{2}$

${ }^{1}$ Centre for Mental Health Research, Research School of Population Health, The Australian National University, Canberra, Australia.

${ }^{2}$ Black Dog Institute, University of New South Wales, Sydney, Australia.

Corresponding author: Philip J. Batterham, 63 Eggleston Road, Acton, ACT 2601, Australia; +61 261251031 (phone); +61 261250733 (fax); philip.batterham@anu.edu.au

Word count: 4823

Key words: adherence, engagement, suicide, internet interventions

Acknowledgements: This study was supported by a grant from the Australian National Health and Medical Research Council (NHMRC; GNT1046317) and forms part of the program of research conducted by the NHMRC Centre of Research Excellence in Suicide Prevention (GNT1042580). The funder does not have any role or ultimate authority in the study design; collection, management, analysis, and interpretation of data; writing of the report; or the decision to submit the report for publication. PJB, ALC, MT and HC are supported by NHMRC fellowships 1158707, 1122544, 1138710 and 1056964. 


\begin{abstract}
Background: The effectiveness of internet-based self-help programs for mental health may be limited by low engagement. Identifying factors associated with engagement in online interventions assists in developing strategies to improve efficacy through greater engagement. The aim of the current study was to identify factors associated with engagement among people with suicidal thoughts who completed an online program.
\end{abstract}

Method: 418 adults with suicidal ideation were recruited online into a randomized controlled trial of a 6-week internet-based self-help program. Program usage for the intervention and active control conditions was measured as the number of logins and modules accessed. Predictors of program usage and between-group differences were examined, including sociodemographic variables, user preferences and mental health status.

Results: Both the control group and the intervention group accessed approximately three modules ( $M=3.1, S D=2.0$ v. $M=2.8, S D=2.1$, respectively), although participants in the intervention group had a significantly higher number of logins (17.3 vs 9.7, $p<0.001$ ). Across both conditions, individuals with more severe suicidal thoughts had better engagement with their respective program. More logins for both programs were also associated with being female, married or in a de-facto relationship, not employed, less severe depression and less willingness to seek help from informal sources.

Limitations: Metrics of adherence may not directly reflect engagement with the program. There may be additional unmeasured factors associated with engagement.

Conclusions: The findings suggest that different engagement strategies may be required depending on sociodemographic and clinical characteristics. Tailoring interventions to at-risk subgroups may optimise health and functional outcomes. 
In the general population, $2.0-3.3 \%$ of adults report suicidal ideation in the past year, with up to $0.6 \%$ reporting a suicide attempt (Borges et al., 2010; Johnston et al., 2009; Kessler et al., 2005). However, most adults experiencing suicidal thoughts or behaviours do not seek professional help (Bruffaerts et al., 2011; Johnston et al., 2009; Luoma et al., 2002). Reasons for this lack of treatment seeking may include stigma or shame, high self-reliance, low suicide literacy, limited access to appropriate services or a lack of perceived need (Han et al., 2018). Many of the identified barriers to help seeking may be overcome by internet-based self-help programs (van Spijker et al., 2014b; van Spijker et al., 2018; Witt et al., 2017). These programs typically provide evidence-based therapy with high-fidelity, in a setting that is free or low-cost, typically anonymous, and available at all times (Musiat and Tarrier, 2014). However, the effectiveness of internet-based programs may be limited by high attrition rates and low engagement (Batterham et al., 2008; Christensen et al., 2009; Donkin et al., 2011; Neil et al., 2009). By identifying the characteristics of individuals who engage less with an intervention, it may be possible to tailor the intervention to better suit their needs.

Engagement with a psychosocial intervention, such as internet-based cognitive behavioural therapy (iCBT), is complex. Unlike medication prescribing, there is limited evidence as to what "dosage" is sufficient and how much variability there is in sufficient dosage between individuals (Donkin et al., 2011). There may be a range of reasons, both negative and positive, which might explain why an individual would discontinue an internet-based intervention, such as lack of apparent improvement, low perceived need for treatment, early remission of symptoms, or poor fit with preferences for treatment delivery (Batterham and Calear, 2017; Batterham et al., 2008; Hilvert-Bruce et al., 2012). There are also challenges in defining indicators of treatment engagement (Donkin et al., 2011). Adherence to modules or 
sessions within an intervention is a commonly-used metric, which captures the proportion of an intervention that the individual is exposed to but may not identify the depth of engagement. Numbers of logins may be a better indicator of how engaged or motivated an individual is, although it is possible to have many logins with minimal exposure to the intervention. It has been suggested that assessing multiple metrics of engagement may provide more robust information on the factors associated with engagement (Donkin et al., 2011). Fortunately, internet interventions typically enable automated collection of multiple indices of engagement.

Research on adherence and engagement with internet interventions has focused on interventions targeting mental and physical health behaviours, and substance use (Christensen et al., 2009; Donkin et al., 2011). However, no studies have yet examined factors influencing engagement with iCBT interventions that target suicidal thoughts and suicidal behaviours. People with suicidal ideation may have particular difficulties in sustaining engagement with a self-guided intervention. For example, suicidal ideation is typically associated with severe mental illness, which can have a considerable negative impact on daily functioning (Batterham et al., 2018; Nock et al., 2009). There is also some evidence that there are specific cognitive impairments associated with suicidal thoughts and behaviours, particularly in executive function, attentional control and working memory (Keilp et al., 2013). High cognitive function may be required to sustain engagement in self-guided psychosocial programs. Despite these barriers to engagement, there remains a large number of people who seek online support for suicidal ideation, including individuals who may be reluctant to engage with health services (van Spijker et al., 2018; Wong et al., 2019). Characterising the types of individuals with suicidal ideation who have the most capacity to engage with internet 
interventions may therefore assist in targeting the delivery of self-guided internet interventions to those most likely to derive benefit.

The present study involved an examination of two indicators of engagement in the Healthy Thinking trial, a randomised controlled trial that investigated the effectiveness of an internetbased self-help therapy program for people experiencing suicidal thoughts. The methods and primary results have been published elsewhere, and indicated that the program was not effective overall, although those who had greater engagement with the program displayed a short-term significant benefit relative to an attentional control (van Spijker et al., 2015; van Spijker et al., 2018). Consequently, identifying the types of individuals who best engage with the program may assist in optimally targeting the program. The current study aimed to identify factors associated with the number of modules accessed and number of logins in both the active intervention and the attention control condition. The active intervention focused on reducing suicidal thinking using therapeutic approaches based on Cognitive Behavioural Therapy (CBT), but also integrating components of other treatment modalities, including Dialectical Behavioural Therapy, problem solving and mindfulness-based approaches. In contrast, the attention control intervention focused on healthy lifestyles. The rationale for examining engagement in both conditions was that there may be general factors that underlie engagement in psychosocial interventions and other health behaviours (such as agreeableness and conscientiousness), but also specific factors that impact on engagement with CBT interventions. iCBT is likely to be more cognitively challenging than a healthy lifestyle intervention. Therefore, the aim of this study was to identify the factors that specifically underlie engagement with iCBT, above and beyond general factors associated with adherence to a non-therapeutic online intervention. The findings from this research may provide insights into how best to engage individuals with suicidal ideation in therapy. 


\section{Methods}

\section{Overview}

This study is a secondary analysis of the Healthy Thinking trial, which was a randomised controlled trial that investigated the effectiveness of an online self-help program for suicidal thoughts. The methods and primary results have been published elsewhere (van Spijker et al., 2015; van Spijker et al., 2018). The current study focuses on the usage of the program. The trial was registered with Australia \& New Zealand Clinical Trials Registry (ACTRN12613000410752) and received ethical approval from the Australian National University Human Research Ethics Committee (protocol 2012/417) and the University of New South Wales (protocol HC13117).

\section{Participants}

Participants were recruited between November 2013 and December 2015 through online media forums including websites, social networking websites, and advertising on search engines. The study portal received 12,474 visits during recruitment, of whom $31 \%(n=3,645)$ completed the screening questionnaire. Two-thirds $(n=2,394)$ of those who completed screening were eligible to participate and 995 participants consented. Participation in the trial required contacting a suicide hotline service to obtain a confidential identification code, which was achieved by $45 \%$ of consenting participants $(n=446)$. Of these participants, $94 \%$ completed the baseline assessments and were randomized into the trial, forming the dataset used in the current analyses $(n=418)$. Eligibility criteria were: age 18 to 65 years, valid email address, access to a reliable internet connection, located in Australia, fluent in English, no history of a diagnosed psychotic disorder, currently experiencing suicidal thoughts, and no suicide attempts in the past month. These were assessed using single, self-report questions. 
No restrictions were placed on the severity of current suicidal thinking or depression. The online program was not supported by human coaching, although reminder emails to complete each module were sent to participants in both the intervention and control conditions. Additional details are provided in the trial protocol (van Spijker et al., 2015) and primary outcomes paper (van Spijker et al., 2018).

\section{Intervention program}

Living with Deadly Thoughts (LwDT) is an adaptation of the 'Leven onder Controle' program ('Living Under Control’), originally developed for a Dutch population (van Spijker et al., 2014). The program is automated, delivered online in six modules, and aims to reduce suicidal thinking severity. The six modules focus on: 1) the nature of repetitive suicidal thinking, 2) emotion regulation, 3) thought identification, 4) thinking patterns, 5) challenging unhelpful beliefs, and 6) relapse prevention. Participants ideally complete one module each week, with subsequent modules becoming available four days after the start of the previous module, regardless of completion. Each module takes approximately 30 minutes to complete, and includes a theory component, a weekly assignment, and practice exercises. Access to referral information via a 'get help now' link was available on every web page.

\section{Control program}

The control program, Living Well, was developed as the comparator to the intervention, and was matched for length, style and format. Living Well focuses on lifestyle information, covering topics such as nutrition, maintaining a healthy environment, preventing heart disease and skin care, with no reference to mental health content. As with the LwDT program, each of the six modules takes approximately 30 minutes to complete and is ideally completed weekly, with each new module becoming available four days after the start of the 
previous module. Access to referral information via a 'get help now' link was also available on every web page.

\section{Measures}

\section{Program usage}

A set of metrics was automatically recorded within the trial to indicate program usage, including the number of logins $(\geq 1)$ and number of the modules accessed (0-6). The number of logins indicates the number of times a participant logged into the website using their unique login details. The six modules were delivered in a fixed sequence. Each module became available four days after the release of the previous module, regardless of completion, and remained available throughout the study period. The module was marked as “accessed" in the system after being clicked to open.

\section{Predictors of program usage}

Predictors of program usage explored in this study were assessed at baseline and included sociodemographic variables, user preferences and mental health status.

Sociodemographic variables included sex, age, education (completed secondary school or above vs others), marital status (being married or de facto vs others), employment (being employed vs others), and living status (metropolitan vs others).

User habits and preferences included items that assessed whether participants were familiar with internet use (Yes/No), whether they had used any mental health services (lifetime: Yes/No), and willingness to seek help from informal and formal sources measured by the General Help Seeking Questionnaire (Wilson et al., 2005). Willingness to seek help from 
informal sources was calculated by averaging the score of the responses of seeking help from four informal sources, including intimate partners, friends (not related to you), parents, and other relatives/family. Willingness to seek help from formal resources was the average score of the responses of seeking help from three formal sources, including mental health professionals, phone helplines and doctor/GPs. Scores on the informal and formal help seeking variables range from 1 to 7 , with higher scores indicating greater willingness to seek help.

Mental health status-related variables included depressive symptoms measured by the Centre for Epidemiological Studies Depression Scale [CES-D (Radloff, 1977); $\alpha=.871$ in the present sample], anxiety assessed by the 7-item Generalised Anxiety Disorder [GAD-7 (Spitzer et al., 2006); $\alpha=.851$ ], severity of suicidal thoughts measured by the Suicidal Ideation Attributes Scale [SIDAS (van Spijker et al., 2014a); $\alpha=.741$ ], and lifetime history of suicide attempts (Yes/No). The CES-D and GAD-7 have previously been demonstrated to be valid and reliable indicators of depression and generalised anxiety in clinical and population-based settings (Radloff, 1977; Spitzer et al., 2006), while the SIDAS reliably differentiates severity of suicidal thinking (van Spijker et al., 2014a).

\section{Analyses}

Differences in participant characteristics between the attention control group and the active intervention group at pre-intervention levels were compared using either $\chi^{2}$ tests or independent sample $t$ tests for categorical and continuous variables respectively, except for number of logins and modules accessed. Poisson regressions were used to compare betweengroup differences and predictors of number of logins, with incidence rate ratios reported. Ordinal multinomial logistic regression models were used to identify predictors of modules 
accessed, with odds ratios reported. The influence of the predictors was assessed firstly using base models including condition (control versus intervention), each individual predictor and the interaction between group and the predictor. Subsequently, significant predictors and variables that significantly interacted with condition (control versus intervention) were included in a multivariate model to characterise differences in predictors of engagement across the two conditions, while adjusting for potentially confounding effects. Significance levels were set at $p<0.05$. All analyses were performed using SPSS v25 (SPSS Inc, Chicago, IL, USA).

\section{Results}

Participant characteristics

The participant flow of this study has been reported in another study (van Spijker et al., 2018). In brief, of the 446 eligible participants recruited to the study, 418 (93.7\%) contacted the Suicide Call Back Service (SCBS) to obtain an identification code, which was an ethical requirement for enrolment in the study. After enrolment, participants were randomised into one of two groups: the control group $(n=211)$ or the intervention group $(n=207)$. No significant difference was found between the two groups on most of the sociodemographic, user preference, and mental health variables (see Table 1), except that the intervention group had more severe suicidal thoughts at pre-intervention than the control group $(p=0.008)$ measured by the SIDAS with a small to medium effect size (Cohen’s $d$ ) of 0.26 .

\section{Program usage}

Both the control group ( $M=3.1$ modules, $S D=2.0$ modules $)$ and the intervention group $(M=2.8$ modules, $S D=2.1$ modules) accessed approximately three modules (50\% of each program), although this small difference was significant $(p=0.041)$. Participants in the intervention 
group had a significantly higher number of logins (17.3 vs 9.7, $p<0.001)$ than those in the control group. On average, participants in the control group logged into the program around three times per accessed module, while the participants in the intervention group logged in around six times per accessed module. Among the 211 participants in the control group, 10 (4.7\%) did not access any of the modules even though they logged into the program more than two times on average $(M=2.6, S D=2.3)$. Seventeen $(8.2 \%)$ participants in the intervention group did not access any of the modules, although they had a mean of 4.8 logins $(S D=4.9)$. Nearly one-third of the participants in the control group ( $n=57,27.0 \%)$ and the intervention group ( $n=65,31.4 \%$ ) accessed only one module, accounting for the highest proportion in terms of number of modules accessed. The second highest proportion came from those who accessed six modules: 49 (23.2\%) participants in the control group and 46 (22.2\%) participants in the intervention group. The sample was combined for subsequent analyses, with adjustment for condition (control vs. intervention) and exploration of potential interaction effects between condition and other predictors to determine whether effects were specific to the active intervention or generic to either internet-based psychosocial intervention.

\section{Predictors of program logins}

Predictors of logins to the programs are presented in Table 2. Three significant interaction effects were observed and included in the model, suggesting differential intervention effects on the basis of education, use of mental health services and past suicide attempt. After adjusting for condition differences and other confounds, participants in the control and intervention conditions were likely to log into their respective online programs more often if they reported being: female (IRR $=0.92,95 \% \mathrm{CI}: 0.86-0.99, p=0.021$ ), educated at above high school level, married or in a de facto relationship (IRR = 1.17, 95\% CI: 1.11-1.24, $p<$ 
0.001), not employed (IRR $=0.88$, 95\% CI: 0.83-0.93, $p<0.001$ ), less willing to seek help from informal sources (IRR $=0.90,95 \%$ CI: 0.88-0.92, $\mathrm{p}<0.001)$, or less familiar with the use of the internet (IRR $=0.76,95 \%$ CI: 0.71-0.82, $p<0.001)$. The relationship with education was complex, with the interaction effect suggesting higher education was associated with fewer logins for the iCBT condition (bachelors or above, IRR $=0.88,95 \%$ CI: 0.81-0.97, $p=0.006$; certificate/diploma associate, IRR $=0.80,95 \%$ CI: 0.72-0.88, $p<$ 0.001 ) but more logins for the control condition (bachelors or above, IRR $=1.40,95 \% \mathrm{CI}$ : 1.23-1.59, $p<0.001$; certificate/diploma associate, IRR $=1.50,95 \%$ CI: 1.31-1.72, $p<$ 0.001). Participants who had previously used mental health services had 35\% higher likelihood (95\% CI: 1.13-1.61) of an additional login than those who had not used mental health services $(p=0.001)$. However, the effect of past mental health service use was significantly greater for the iCBT program than for the control program $(p=0.008)$.

In terms of the influence of mental health status on program logins, relative to participants who reported one lifetime suicide attempt, participants who had reported more than one lifetime suicide attempts were significantly more likely to have additional logins. Specifically, after account for the significant interaction with condition, participants with multiple suicide attempts in the control condition were 36\% more likely [95\% CI: 1.21-1.54] to have an additional login to their program $(p<0.001)$, but participants with multiple suicide attempts in the intervention group were $21 \%$ less likely [95\% CI: $0.72-0.87$ ] to have an additional login to their program $(p<0.001)$, after adjusting for condition differences and other potential confounders. No significant difference was found between participants who reported one lifetime suicide attempt and those without lifetime attempt on program logins. Meanwhile, participants who were at high risk of suicidal behaviours indicated by having a SIDAS score of at least 21 (van Spijker et al., 2014a) were 22\% more likely [95\% CI: 1.15- 
1.30] than those who were at low risk (SIDAS =1) to have an additional login for both programs $(p<0.001)$.

No between-group difference was found on the influence of depressive symptoms on program logins measured by CESD. Individuals who had a CESD score of $45{\text { ( } 75^{\text {th }}}^{\text {th }}$ percentile) were $42 \%$ less likely [95\% CI: $0.53-0.65$ ] to have an additional login for both programs than those had a CESD score of $15\left(25^{\text {th }}\right.$ percentile $)(p<0.001)$. Although anxiety symptoms were associated with fewer logins in the base model, the influence was not sustained in the multivariate model.

\section{Predictors of the number of modules accessed}

Predictors of the number of modules accessed are presented in Table 3. Three significant interaction effects were observed and included in the model, suggesting differential intervention effects on the basis of education, marital status, and location. After adjustment for condition differences and other confounders, participants who had a bachelor degree or above had $40 \%$ greater odds [95\% CI: $1.03-1.90$ ] of accessing an additional module of the compared to those who had high school or less education $(p=0.034)$. However, the significant interaction effect suggested differential relationships, with greater education leading to a small reduction in module access in the intervention condition $(p=0.043)$. Meanwhile, those who were married or in a de facto relationship had 76\% greater odds [95\% CI: 1.38-2.24] of accessing one more module of the control program $(p<0.001)$, although there was no significant influence of relationship status on the number of modules accessed in the intervention program. No other demographics or user preferences were found to have a significant influence on module access in the multivariate model. 
Regarding mental health characteristics, individuals who were at high risk of suicidal behaviours (SIDAS $\geq 21$ ) had 30\% greater odds [95\% CI: 1.11-1.52] of accessing on additional module of either program, compared to those who were at low risk (SIDAS=1; $p<$ 0.001). No significant effect of depressive or anxious symptoms was found on module access in either the base or multivariate models.

\section{Discussion}

Better understanding of engagement with internet interventions may enable optimisation of these programs to ensure that they are appropriately targeted and tailored to the needs of end users. The current study was the first to investigate factors associated with engagement to iCBT among individuals with suicidal ideation. The study included comparison to an attention control condition with random allocation, which enabled differentiation between factors that were specifically associated with iCBT engagement and factors more generally associated with an individual's propensity to engage in a health intervention. The findings indicate a number of key considerations for future design of interventions to reduce suicidal thinking, as factors related to the demographics, user preferences and mental health status were related to the number of times a participant logged into the intervention and the number of modules they accessed. Many of the factors identified were generically associated with engagement to either intervention, which suggests that common factors may underlie engagement in psychosocial interventions for people experiencing suicidal thinking, irrespective of the content of the intervention. Nevertheless, there were also some specific factors identified, which may suggest certain subgroups had greater challenges with a CBT intervention, or that the lifestyle intervention (which are not typically effective for reducing suicidal thinking) may have impacted on participant motivations to continue to progress through the intervention. 
Males had fewer logins than females, although they accessed a similar number of modules. Males are typically harder to reach in trials of mental health interventions than females, partly because they are less likely to experience suicidal ideation but also because they tend to engage less with psychosocial interventions, similarly to traditional therapy (Burgess et al., 2009). Efforts to better tailor interventions to males have had mixed success (Mocarski and Butler, 2016) and is an important area for further exploration (Seidler et al., 2018). In contrast to a previous review (Christensen et al., 2009), there was an association of older age with more logins in the base model, although this effect was not significant after adjustment for other characteristics. This finding suggests that internet interventions for suicide prevention are not simply the domain of young people, but may be similarly engaging for older adults. It could also be the case that older adults were less efficient at using the intervention, so required more logins to progress through the intervention.

The complex effect of higher levels of education on engagement may suggest that those with less education may have engaged more poorly with the attention-control (lifestyle) intervention than with the iCBT intervention. It is possible that the control materials were challenging for participants with less education, while the iCBT program had less appeal for those with greater education. The development of content that is accessible across varying levels of educational background can be challenging. Persuasive system design, use of videos and interactive elements may increase the appeal of an intervention across a broader audience (Kelders et al., 2012). Those who were employed tended to log into the website less frequently, an unexpected finding which may reflect time constraints. Participants who were married or in a de facto relationship tended to login more frequently within online modules. This finding may suggest that social support can facilitate engagement with online 
interventions (Kelders et al., 2012), that family responsibilities may increase an individual's motivation to get better, or that relationship breakdown may lead to deterioration and additional challenges in adhering to an online program (Kazan et al., 2016). However, the effects of marital status were only significantly greater in the attention control intervention than the iCBT intervention, suggesting the additional support associated with having a partner may have limited impact on engagement with more demanding therapeutic interventions. Future research examining the impact of relationship quality on engagement may shed further light on these findings.

Although user preferences were associated with logins, they had no significant relationships with module access. Less familiarity with the internet was associated with more logins, which may indicate that the novelty of internet-based programs may be greater for those who do not typically use the internet, leading them to engage more often and possibly more deeply with the content. However, this finding may also reflect greater inefficiency in accessing the intervention among those with low familiarity, as the increased logins did not lead to engagement with additional modules. Although the effect of familiarity was independent of age, there may be distinct patterns of internet use that are associated with older age (Tennant et al., 2015). Use of mental health services was associated with more logins. This finding is complex, as use of services might reflect severity or chronicity of mental illness or greater readiness for change. Nonetheless, the finding indicates that individuals who have received support from professional services might be more inclined to engage with an internet-based intervention, although again, this engagement did not translate into accessing additional modules. Moreover, the effect was significantly greater for the active iCBT intervention, which may suggest that existing exposure to CBT might facilitate engagement with an online CBT program. In contrast, those naïve to CBT principles may have struggled more with the 
content of the active program. Greater willingness to engage with informal resources was associated with fewer logins, which suggests that people who are willing to engage socially with others to support their mental health (offline) may be less reliant on online support.

Severity of mental health problems had a complex relationship with intervention engagement. More severe suicidal ideation was associated with more logins and module access, suggesting that those struggling more with suicidal thoughts may have found the intervention to provide some degree of support or may have been more aware of their need for help. However, more severe depression or anxiety symptoms was associated with fewer logins, suggesting that a high severity of mental health symptoms may disrupt an individual's ability to engage with an internet intervention. Another possible explanation is that people with high levels of depression and anxiety may not have found the content as relevant to them and their symptoms than those with high levels of suicidal ideation, since the focus of the content was on reducing suicidal thinking rather than depression or anxiety specific. This explanation is supported by previous research that has found interventions directly targeting a disorder are often more effective on that disorder than interventions indirectly targeting them (Meerwijk et al., 2016). This finding is also consistent with a previous review that found severe depression symptoms to reduce adherence to online interventions (Christensen et al., 2009). The effect of suicidal ideation on greater engagement appears to be distinct and suggests that the desire for support among people experiencing suicidal thoughts may overcome some of the barriers to engagement. However, relationships with suicidal behaviour differed across the two interventions, suggesting that history of suicidal behaviour might increase engagement with a lifestyle intervention but decrease engagement with a CBT intervention. This finding suggests that individuals with more complex or severe mental health histories 
may have been familiar with structured therapeutic interventions such as CBT, but more motivated to complete the lifestyle intervention.

There are a number of limitations to the current study. Although we used two measures of engagement, there are other potential metrics, such as time spent on each page, number of activities completed within modules, or self-reported ratings of engagement. No metric of engagement is perfect - for example, more logins may equate to less efficient use of the intervention. Exploring additional metrics of engagement might better elucidate the factors underlying intervention engagement. The sample was restricted to individuals with suicidal ideation but no recent suicide plan or attempt, and included over-representation of some population groups (e.g., females), which may limit the generalisability of the findings to other populations. The intervention group had more severe suicidal ideation at baseline than the control group, so differences in engagement may be related to this minor disparity. Finally, there is likely to be a range of other factors related to engagement, such as preferences, abilities, health and motivation, that may also influence the degree to which an individual engages with an intervention. Additional clinical factors associated with suicidal ideation, such as hopeless and anhedonia, may impact an individual's ability to engage. There would be value in further exploring these factors in future research, particularly modifiable factors that could be directly targeted prior to the commencement of an online intervention. Further research is currently exploring the use of engagement facilitation interventions, that may reduce some of the barriers to engagement in online programs (Batterham et al., 2019).

In conclusion, the present study identified a number of factors associated with engagement to internet interventions among people experiencing suicidal ideation. Most of these factors were associated with engagement generically, and not specific to the CBT intervention. 
Males, people with limited education, people in employment and people with limited social support tended to engage less with the interventions. Greater familiarity with the internet and greater willingness to seek help elsewhere were also associated with poorer engagement. A complex relationship between mental health and engagement emerged, such that more severe suicidal ideation and less severe depression/anxiety symptoms were associated with greater engagement. Designing interventions that meet the diverse needs of the population poses a challenge for researchers and an opportunity for improving outcomes. Understanding the mechanisms underlying engagement in treatment may lead to more appropriate use of services that fit the individual. The use of qualitative research methods, co-design principles and engagement facilitation interventions may lead to a better understanding of the barriers to engagement and the development interventions that are more engaging and impactful. By tailoring interventions to at-risk subgroups that engage poorly, and targeting interventions to groups most likely to benefit, internet interventions for suicidal individuals may be optimised to improve health and functional outcomes. 


\section{References}

Batterham, P.J., Calear, A.L., 2017. Preferences for Internet-Based Mental Health Interventions in an Adult Online Sample: Findings From an Online Community Survey. JMIR Ment Health 4, e26.

Batterham, P.J., Calear, A.L., Christensen, H., Carragher, N., Sunderland, M., 2018. Independent Effects of Mental Disorders on Suicidal Behavior in the Community. Suicide Life Threat Behav 48, 512-521.

Batterham, P.J., Calear, A.L., Sunderland, M., Kay-Lambkin, F., Farrer, L.M., Gulliver, A., 2019. A brief intervention to increase uptake and adherence of an online program for depression and anxiety: Protocol for the Enhancing Engagement with Psychosocial Interventions (EEPI) Randomized Controlled Trial. Contemp Clin Trials 78, 107-115. Batterham, P.J., Neil, A.L., Bennett, K., Griffiths, K.M., Christensen, H., 2008. Predictors of adherence among community users of a cognitive behavior therapy website. Patient Prefer Adherence 2, 97-105.

Borges, G., Nock, M.K., Haro Abad, J.M., Hwang, I., Sampson, N.A., Alonso, J., Andrade, L.H., Angermeyer, M.C., Beautrais, A., Bromet, E., Bruffaerts, R., de Girolamo, G., Florescu, S., Gureje, O., Hu, C., Karam, E.G., Kovess-Masfety, V., Lee, S., Levinson, D., Medina-Mora, M.E., Ormel, J., Posada-Villa, J., Sagar, R., Tomov, T., Uda, H., Williams, D.R., Kessler, R.C., 2010. Twelve-month prevalence of and risk factors for suicide attempts in the World Health Organization World Mental Health Surveys. J Clin Psychiatry 71, 16171628.

Bruffaerts, R., Demyttenaere, K., Hwang, I., Chiu, W.T., Sampson, N., Kessler, R.C., Alonso, J., Borges, G., de Girolamo, G., de Graaf, R., Florescu, S., Gureje, O., Hu, C., Karam, E.G., Kawakami, N., Kostyuchenko, S., Kovess-Masfety, V., Lee, S., Levinson, D., Matschinger, H., Posada-Villa, J., Sagar, R., Scott, K.M., Stein, D.J., Tomov, T., Viana, 
M.C., Nock, M.K., 2011. Treatment of suicidal people around the world. Br J Psychiatry 199, 64-70.

Burgess, P.M., Pirkis, J.E., Slade, T.N., Johnston, A.K., Meadows, G.N., Gunn, J.M., 2009. Service use for mental health problems: findings from the 2007 National Survey of Mental Health and Wellbeing. Aust N Z J Psychiatry 43, 615-623.

Christensen, H., Griffiths, K.M., Farrer, L., 2009. Adherence in internet interventions for anxiety and depression. J Med Internet Res 11, e13.

Donkin, L., Christensen, H., Naismith, S.L., Neal, B., Hickie, I.B., Glozier, N., 2011. A systematic review of the impact of adherence on the effectiveness of e-therapies. $\mathrm{J}$ Med Internet Res 13, e52.

Han, J., Batterham, P.J., Calear, A.L., Randall, R., 2018. Factors Influencing Professional Help-Seeking for Suicidality. Crisis 39, 175-196.

Hilvert-Bruce, Z., Rossouw, P.J., Wong, N., Sunderland, M., Andrews, G., 2012. Adherence as a determinant of effectiveness of internet cognitive behavioural therapy for anxiety and depressive disorders. Behav Res Ther 50, 463-468.

Johnston, A.K., Pirkis, J.E., Burgess, P.M., 2009. Suicidal thoughts and behaviours among Australian adults: findings from the 2007 National Survey of Mental Health and Wellbeing. Aust N Z J Psychiatry 43, 635-643.

Kazan, D., Calear, A.L., Batterham, P.J., 2016. The impact of intimate partner relationships on suicidal thoughts and behaviours: A systematic review. J Affect Disord 190, 585-598. Keilp, J.G., Gorlyn, M., Russell, M., Oquendo, M.A., Burke, A.K., Harkavy-Friedman, J., Mann, J.J., 2013. Neuropsychological function and suicidal behavior: attention control, memory and executive dysfunction in suicide attempt. Psychol Med 43, 539-551. 
Kelders, S.M., Kok, R.N., Ossebaard, H.C., Van Gemert-Pijnen, J.E., 2012. Persuasive system design does matter: a systematic review of adherence to web-based interventions. J Med Internet Res 14, e152.

Kessler, R.C., Berglund, P., Borges, G., Nock, M., Wang, P.S., 2005. Trends in suicide ideation, plans, gestures, and attempts in the United States, 1990-1992 to 2001-2003. JAMA 293, 2487-2495.

Luoma, J.B., Martin, C.E., Pearson, J.L., 2002. Contact with mental health and primary care providers before suicide: a review of the evidence. Am J Psychiatry 159, 909-916.

Meerwijk, E.L., Parekh, A., Oquendo, M.A., Allen, I.E., Franck, L.S., Lee, K.A., 2016. Direct versus indirect psychosocial and behavioural interventions to prevent suicide and suicide attempts: a systematic review and meta-analysis. Lancet Psychiatry 3, 544-554. Mocarski, R., Butler, S., 2016. A Critical, Rhetorical Analysis of Man Therapy: The Use of Humor to Frame Mental Health as Masculine. Journal of Communication Inquiry 40, 128144.

Musiat, P., Tarrier, N., 2014. Collateral outcomes in e-mental health: a systematic review of the evidence for added benefits of computerized cognitive behavior therapy interventions for mental health. Psychol Med 44, 3137-3150.

Neil, A.L., Batterham, P., Christensen, H., Bennett, K., Griffiths, K.M., 2009. Predictors of adherence by adolescents to a cognitive behavior therapy website in school and communitybased settings. J Med Internet Res 11, e6.

Nock, M.K., Hwang, I., Sampson, N., Kessler, R.C., Angermeyer, M., Beautrais, A., Borges, G., Bromet, E., Bruffaerts, R., de Girolamo, G., de Graaf, R., Florescu, S., Gureje, O., Haro, J.M., Hu, C., Huang, Y., Karam, E.G., Kawakami, N., Kovess, V., Levinson, D., PosadaVilla, J., Sagar, R., Tomov, T., Viana, M.C., Williams, D.R., 2009. Cross-national analysis of 
the associations among mental disorders and suicidal behavior: findings from the WHO World Mental Health Surveys. PLoS Med 6, e1000123.

Radloff, L.S., 1977. A Self-Report Depression Scale for Research in the General Population Applied Psychological Measurement 1, 385-401.

Seidler, Z.E., Rice, S.M., Ogrodniczuk, J.S., Oliffe, J.L., Dhillon, H.M., 2018. Engaging Men in Psychological Treatment: A Scoping Review. Am J Mens Health 12, 1882-1900.

Spitzer, R.L., Kroenke, K., Williams, J.B., Lowe, B., 2006. A brief measure for assessing generalized anxiety disorder: the GAD-7. Arch Intern Med 166, 1092-1097.

Tennant, B., Stellefson, M., Dodd, V., Chaney, B., Chaney, D., Paige, S., Alber, J., 2015. eHealth literacy and Web 2.0 health information seeking behaviors among baby boomers and older adults. J Med Internet Res 17, e70.

van Spijker, B.A., Batterham, P.J., Calear, A.L., Farrer, L., Christensen, H., Reynolds, J., Kerkhof, A.J., 2014a. The suicidal ideation attributes scale (SIDAS): Community-based validation study of a new scale for the measurement of suicidal ideation. Suicide Life Threat Behav 44, 408-419.

van Spijker, B.A., Calear, A.L., Batterham, P.J., Mackinnon, A.J., Gosling, J.A., Kerkhof, A.J., Solomon, D., Christensen, H., 2015. Reducing suicidal thoughts in the Australian general population through web-based self-help: study protocol for a randomized controlled trial. Trials 16, 62.

van Spijker, B.A., van Straten, A., Kerkhof, A.J., 2014b. Effectiveness of online self-help for suicidal thoughts: results of a randomised controlled trial. PLoS One 9, e90118.

van Spijker, B.A., Werner-Seidler, A., Batterham, P.J., Mackinnon, A., Calear, A.L., Gosling, J.A., Reynolds, J., Kerkhof, A.J., Solomon, D., Shand, F., Christensen, H., 2018. Effectiveness of a Web-Based Self-Help Program for Suicidal Thinking in an Australian Community Sample: Randomized Controlled Trial. J Med Internet Res 20, e15. 
Wilson, C.J., Deane, F.P., Ciarrochi, J., Rickwood, D., 2005. Measuring Help-Seeking Intentions: Properties of the General Help-Seeking Questionnaire. Canadian Journal of Counselling 39, 15-28.

Witt, K., Spittal, M.J., Carter, G., Pirkis, J., Hetrick, S., Currier, D., Robinson, J., Milner, A., 2017. Effectiveness of online and mobile telephone applications ('apps') for the selfmanagement of suicidal ideation and self-harm: a systematic review and meta-analysis. BMC Psychiatry 17, 297.

Wong, Q.J., Werner-Seidler, A., Torok, M., van Spijker, B., Calear, A.L., Christensen, H., 2019. Service Use History of Individuals Enrolling in a Web-Based Suicidal Ideation Treatment Trial: Analysis of Baseline Data. JMIR Ment Health 6, e11521. 
Table 1. Characteristics of participants and engagement metrics in the attention control group (Living Well) and the active intervention group (Living with Deadly Thoughts)

\begin{tabular}{|c|c|c|c|}
\hline & $\begin{array}{l}\text { Attention control } \\
\quad(\mathrm{n}=211)\end{array}$ & $\begin{array}{l}\text { Active intervention } \\
\qquad(\mathrm{n}=207)\end{array}$ & $p$ \\
\hline \multicolumn{4}{|l|}{ Demographics } \\
\hline Female, n (\%) & $163(78.0 \%)$ & $160(77.3 \%)$ & 0.992 \\
\hline Age, years, mean (SD) & $41.7(11.9)$ & 39.5 (11.9) & 0.060 \\
\hline Education n (\%) & & & 0.917 \\
\hline High school or less & $42(19.9 \%)$ & $38(18.4 \%)$ & \\
\hline Certificate/diploma/associate degree & $68(32.2 \%)$ & $67(32.4 \%)$ & \\
\hline Bachelor degree or higher & $101(47.9 \%)$ & $102(50.2 \%)$ & \\
\hline Marital status (married or de facto), n (\%) & $73(34.6 \%)$ & $87(42.0 \%)$ & 0.118 \\
\hline Employment (employed), n (\%) & $127(60.2 \%)$ & $121(58.5 \%)$ & 0.718 \\
\hline Metropolitan, n (\%) & $126(59.7 \%)$ & 127 (61.4\%) & 0.732 \\
\hline \multicolumn{4}{|l|}{ User preferences } \\
\hline Being familiar with internet use, n (\%) & $174(82.5 \%)$ & $174(84.1 \%)$ & 0.663 \\
\hline Having used mental health service, $\mathrm{n}(\%)$ & $202(95.7 \%)$ & $201(97.1 \%)$ & 0.453 \\
\hline Willingness to seek help from informal resources, mean $(S D)$ & $2.5(1.4)$ & $2.5(1.3)$ & 0.967 \\
\hline Willingness to seek help from formal resources, mean (SD) & $4.1(1.6)$ & $4.0(1.5)$ & 0.727 \\
\hline \multicolumn{4}{|l|}{ Mental health status } \\
\hline Reported lifetime suicide attempt(s), n (\%) & & & 0.666 \\
\hline No attempt & $101(47.9 \%)$ & $90(43.5 \%)$ & \\
\hline One attempt & $43(20.4 \%)$ & $46(22.2 \%)$ & \\
\hline More than one attempts & $67(31.8 \%)$ & $71(34.3 \%)$ & \\
\hline Severity of suicidal thoughts - SIDAS, mean (SD) & $25.4(10.5)$ & $28.0(9.7)$ & 0.008 \\
\hline Severity of depression - CESD, mean (SD) & $39.7(9.7)$ & $40.8(9.5)$ & 0.243 \\
\hline Severity of anxiety - GAD7, mean (SD) & $13.1(5.1)$ & $13.4(5.0)$ & 0.602 \\
\hline \multicolumn{4}{|l|}{ Engagement } \\
\hline Number of logins, mean (SD) & $9.7(9.1)$ & $17.3(17.1)$ & $<0.001$ \\
\hline Modules accessed, mean (SD) & $3.1(2.0)$ & $2.8(2.1)$ & 0.041 \\
\hline
\end{tabular}

Note. Bold, $p<0.05$ 


\begin{tabular}{|c|c|c|c|c|c|c|}
\hline & \multicolumn{3}{|c|}{ Base model - single predictor* } & \multicolumn{3}{|c|}{ Multiple predictors } \\
\hline & $I R R$ & $95 \%$ CI & $p$ & $I R R$ & $95 \%$ CI & $p$ \\
\hline \multicolumn{7}{|l|}{ Condition } \\
\hline Attention control vs active intervention & 0.56 & $0.53-0.60$ & $<0.001$ & 0.31 & $0.26-0.37$ & $<\mathbf{0 . 0 0 1}$ \\
\hline \multicolumn{7}{|l|}{ Demographics } \\
\hline Male vs female & 0.87 & $0.81-0.95$ & 0.001 & 0.92 & 0.86-0.99 & 0.021 \\
\hline Older age & 1.01 & $1.00-1.01$ & $<0.001$ & 1.00 & $1.00-1.00$ & 0.838 \\
\hline \multicolumn{7}{|l|}{ Education } \\
\hline Bachelors or above vs high school or less & 0.83 & $0.77-0.91$ & $<0.001$ & 0.88 & 0.81-0.97 & 0.006 \\
\hline Certificate/diploma associate vs high school or less & 0.79 & $0.72-0.87$ & $<0.001$ & 0.80 & $0.72-0.88$ & $<0.001$ \\
\hline \multicolumn{7}{|l|}{ Education $\times$ condition (control vs intervention) } \\
\hline Bachelors or above vs high school or less & 1.48 & 1.27-1.72 & $<0.001$ & 1.59 & 1.36-1.85 & $<0.001$ \\
\hline Certificate/diploma associate vs high school or less & 1.74 & $1.48-2.04$ & $<0.001$ & 1.88 & $1.60-2.22$ & $<0.001$ \\
\hline Married or de facto vs others & 1.14 & $1.07-1.22$ & $<0.001$ & 1.17 & 1.11-1.24 & $<0.001$ \\
\hline Employed vs others & 0.88 & $0.82-0.94$ & $<0.001$ & 0.88 & 0.83-0.93 & $<0.001$ \\
\hline Living in metropolitan vs others & 1.00 & $0.93-1.07$ & 0.908 & l & / & l \\
\hline \multicolumn{7}{|l|}{ User preferences } \\
\hline Familiar with internet use & 0.75 & $0.69-0.81$ & $<0.001$ & 0.76 & 0.71-0.82 & $<0.001$ \\
\hline Used mental health services & 1.36 & 1.14-1.61 & $<0.001$ & 1.35 & 1.13-1.61 & 0.001 \\
\hline Used mental health services $\times$ condition & 0.61 & $0.46-0.81$ & 0.001 & 0.67 & $0.50-0.90$ & 0.008 \\
\hline Willingness to seeking help from informal resources & 0.90 & $0.88-0.93$ & $<0.001$ & 0.90 & 0.88-0.92 & $<0.001$ \\
\hline Willingness to seeking help from formal resources & 0.96 & $0.94-0.98$ & $<0.001$ & 1.00 & $0.98-1.02$ & 0.923 \\
\hline \multicolumn{7}{|l|}{ Mental health status } \\
\hline \multicolumn{7}{|l|}{ Lifetime suicide attempt(s) } \\
\hline No attempt vs one attempt & 0.89 & 0.82-0.97 & 0.005 & 0.94 & $0.86-1.03$ & 0.179 \\
\hline More than one attempt vs one attempt & 0.83 & $0.76-0.91$ & $<0.001$ & 0.79 & $0.72-0.87$ & $<\mathbf{0 . 0 0 1}$ \\
\hline \multicolumn{7}{|l|}{ Lifetime suicide attempt(s) $\times$ condition } \\
\hline No attempt vs one attempt & 1.05 & $0.91-1.22$ & 0.511 & 1.01 & $0.87-1.18$ & 0.863 \\
\hline More than one attempt vs one attempt & 1.70 & 1.46-1.97 & $<0.001$ & 1.72 & $1.48-2.00$ & $<0.001$ \\
\hline
\end{tabular}


More severe suicidal thoughts - SIDAS

More severe depression - CESD

1.00-1.01

0.99-0.99

$<0.001$

1.01

1.01-1.01

$<0.001$

More severe anxiety - GAD7

$0.99<0.99-0.99 \quad<0.001$

0.98-0.99

$<0.001$

1.00

0.98-0.99

$<0.001$

Note. *The base models include condition, the individual predictor variable and interaction between condition and predictor variable. The multivariate model includes condition, predictors that were significant in the base models, and significant interactions. Bold, $p<0.05$. 


\begin{tabular}{|c|c|c|c|c|c|c|}
\hline & \multicolumn{3}{|c|}{ Base model - single predictor* } & \multicolumn{3}{|c|}{ Multiple predictors } \\
\hline & OR & $95 \%$ CI & $p$ & OR & $95 \%$ CI & $p$ \\
\hline \multicolumn{7}{|l|}{ Condition } \\
\hline Attention control vs active intervention & 1.18 & 1.01-1.38 & 0.041 & 0.78 & $0.50-1.20$ & 0.253 \\
\hline \multicolumn{7}{|l|}{ Demographics } \\
\hline Male vs female & 0.87 & $0.67-1.14$ & 0.309 & / & / & l \\
\hline Older age & 0.99 & $0.98-1.00$ & 0.064 & / & / & / \\
\hline \multicolumn{7}{|l|}{ Education } \\
\hline Bachelors or above vs high school or less & 1.33 & $0.98-1.80$ & 0.067 & 1.40 & 1.03-1.90 & 0.034 \\
\hline Certificate/diploma associate vs high school or less & 0.84 & $0.61-1.17$ & 0.314 & 0.84 & $0.60-1.18$ & 0.322 \\
\hline \multicolumn{7}{|l|}{ Education $\times$ condition (control vs intervention) } \\
\hline Bachelors or above vs high school or less & 1.01 & $0.66-1.55$ & 0.947 & 0.90 & $0.58-1.38$ & 0.615 \\
\hline Certificate/diploma associate vs high school or less & 1.72 & $1.09-2.71$ & 0.019 & 1.61 & 1.01-2.57 & 0.043 \\
\hline Married or de facto vs others & 1.05 & $0.84-1.32$ & 0.656 & 1.06 & $0.84-1.33$ & 0.624 \\
\hline Married or de facto vs others $\times$ condition & 1.69 & 1.22-2.34 & 0.002 & 1.66 & 1.19-2.32 & 0.003 \\
\hline Employed vs others & 0.82 & $0.66-1.03$ & 0.090 & / & l & / \\
\hline Living in metropolitan vs others & 0.94 & $0.75-1.18$ & 0.579 & 0.90 & $0.71-1.15$ & 0.407 \\
\hline Living in metropolitan vs others $\times$ condition & 1.47 & 1.06-2.02 & 0.019 & 1.37 & $0.98-1.91$ & 0.066 \\
\hline \multicolumn{7}{|l|}{ User preferences } \\
\hline Familiar with internet use & 1.18 & $0.87-1.60$ & 0.296 & 1 & l & 1 \\
\hline Used mental health services & 1.58 & $0.81-3.09$ & 0.184 & / & / & / \\
\hline Willingness to seeking help from informal resources & 0.92 & $0.85-1.00$ & 0.062 & / & / & / \\
\hline Willingness to seeking help from formal resources & 0.96 & $0.89-1.03$ & 0.256 & / & l & / \\
\hline \multicolumn{7}{|l|}{ Mental health status } \\
\hline \multicolumn{7}{|l|}{ Lifetime suicide attempt(s) } \\
\hline No attempt vs one attempt & 0.99 & $0.74-1.32$ & 0.948 & / & / & l \\
\hline More than one attempt vs one attempt & 1.00 & $0.74-1.35$ & 0.991 & l & / & / \\
\hline More severe suicidal thoughts - SIDAS & 1.01 & 1.00-1.02 & 0.038 & 1.01 & 1.01-1.02 & 0.001 \\
\hline More severe depression - CESD & 1.00 & $0.99-1.01$ & 0.675 & / & / & / \\
\hline
\end{tabular}


Note. ${ }^{*}$ The base models include condition, the individual predictor variable and interaction between condition and predictor variable. The multivariate model includes condition, predictors that were significant in the base models, and significant interactions. Bold, $p<0.05$. 